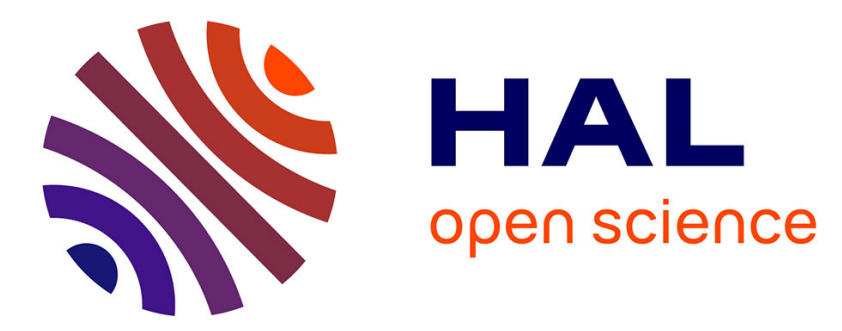

\title{
Interpersonal comparisons of utility in bargaining: evidence from a transcontinental ultimatum game
}

Romina Boarini, Jean-François Laslier, Stéphane Robin

\section{To cite this version:}

Romina Boarini, Jean-François Laslier, Stéphane Robin. Interpersonal comparisons of utility in bargaining: evidence from a transcontinental ultimatum game. Theory and Decision, 2009, 67 (4), pp 341-373. 10.1007/s11238-009-9135-5 . halshs-00451566

\section{HAL Id: halshs-00451566 \\ https://shs.hal.science/halshs-00451566}

Submitted on 18 Feb 2011

HAL is a multi-disciplinary open access archive for the deposit and dissemination of scientific research documents, whether they are published or not. The documents may come from teaching and research institutions in France or abroad, or from public or private research centers.
L'archive ouverte pluridisciplinaire $\mathbf{H A L}$, est destinée au dépôt et à la diffusion de documents scientifiques de niveau recherche, publiés ou non, émanant des établissements d'enseignement et de recherche français ou étrangers, des laboratoires publics ou privés. 


\title{
Interpersonal Comparisons of Utility in Bargaining:
}

\author{
Evidence from a Transcontinental Ultimatum Game ${ }^{1}$
}

\author{
Romina BOARINI² (OECD, Paris), \\ Jean-François LASLIER ${ }^{3}$ (Ecole Polytechnique, Paris), \\ and Stéphane RoBIN ${ }^{4}$ (GATE, Université Lyon 2)
}

Revision, February 2009

\footnotetext{
${ }^{1}$ We would like to thank the Planning Unit of the Indian Statistical Institute (New Delhi) and the Delhi School of Economics School for providing the logistics for the experiments, and especially Arunava Sen, Pulin Nayak, Dipjyoti Majumdar and Puru Vashishtha. Thanks also to Peter Martinsson for his help and assistance. We gratefully acknowledge the financial support of the "GDR d'Economie Cognitive" of the CNRS.

${ }^{2}$ Romina Boarini, OCDE, 2 rue André Pascal, 75775 Paris cedex 16, France. boarini@oecd.org.

${ }_{3}^{3}$ Jean-François Laslier, Département d'Economie, École Polytechnique, 91128 Palaiseau, France. jean-francois.laslier@polytechnique.edu

${ }^{4}$ Stéphane Robin, GATE, 93 chemin des Mouilles, 69131 Ecully, France. robin@gate.cnrs.fr.
} 


\begin{abstract}
:
This paper presents the experimental results of a "Transcontinental Ultimatum Game" implemented between India and France. We use a standard ultimatum game, but in one treatment Indian subjects made offers to French subjects (ItoF treatment) and, in another treatment, French subjects made offers to Indian subjects (FtoI treatment). We observed that FtoI treatment bargaining mostly ended up with unequal splits of money in favor of French, while nearly equal splits were the most frequent outcome in ItoF treatment interactions.

The experimental results are organized through a standard social reference model, modified for taking into account the different marginal value of money for bargainers. In our model bargaining is driven by relative standings comparisons between players, occurring in terms of real earnings (that is monetary earnings corrected for a purchasing power factor) obtained in the game. The norm of equity behind the equalization of real earnings is called local equity norm, and contrasted to a global equity norm which would encompass the wealth of players beyond the game. According to what we observed, no beyond-game concern seems to be relevantly endorsed by subjects.
\end{abstract}

Keywords: Interpersonal Comparisons of Utility, Fairness, Bargaining experiment, Ultimatum Game
JEL code:
A15, C70, C91, D63 


\section{Introduction}

\section{What is an "equitable share"?}

Unlike most normative theories of justice, positive economic theory has traditionally done without interpersonal comparisons of utilities. Empirical work however greatly challenges this received view and highlights that, when justice norms and fairness concerns influence behaviors, an accurate positive theory needs to embody interpersonal comparisons of utilities. This comparison always rests on some underlying notion of equally or equity. But what is to be equalized is a matter of debate (Sen, 1992) and the notion of equity has to be refined. While relative standing comparisons models (Charness and Rabin, 2002, Fehr and Schmidt, 1999) account for bargaining interactions driven by fairness consideration, they usually rest on the assumption of equal marginal value of money. This paper mainly aims at relaxing this assumption and raises the question of what are the relevant interpersonal comparisons of utilities in an experimental setting when money is differently worth to players. Even in such a simple context, the question of what is an "equitable" outcome is not obvious, and various views of what is a practical justice implies providing different answers. This paper considers the three following alternative views ${ }^{5}$ :

- Along the baseline view, inter-personal comparisons of well-being do not have any role in shaping distribution. For instance, if a certain quantity of a divisible good has to be divided between two individuals they should each receive half of it, whoever they are. Call this view "Formal equity".

- Along a second view every occasion should be used to reduce as much as possible inequalities. For instance if a (divisible) good has to be divided between a poor and a rich individual, the largest part should a priori be given to the poor one. Interpersonal comparisons of utilities encompass the whole situation of the individuals and not only the local gain obtained from the interaction. This view can be referred as "Compensation" or "Global equity".

\footnotetext{
${ }^{5}$ The first view is a standard view in theories of distributive justice, while the second and the third ones, although partly related to the conceptions of local and global equity (Elster, 1993), are specifically introduced and discussed in this paper with respect to our experimental setting.
} 
- Finally, a third view recommends the equalization of the local benefits that individuals draw from a given situation. It relies on inter-personal comparisons of these benefits, and only of these. For instance, suppose that a (divisible) good has to be divided between two persons and that, from every unit of the good, the first person draws a lower benefit than the second; then the first person should receive more units of the good that the second. Call this view "Local equity".

The goal of this paper is to test these three competing views. To that aim, we designed and ran a bargaining laboratory experiment, a standard ultimatum game, where rich and poor subjects played against each other and where the stake over which subjects bargained was differently worth to them.

The participants were drawn from countries living under different economic conditions and the diversity was controlled along two dimensions: the income and the purchasing power. With respect to the first point, we chose a high level of income country (France) and a low level of income country (India). With respect to the second point (real value of money), the bargaining was done in US dollars and actual exchange rates were used to convert dollar payoffs to final pay-offs (Indian and French subjects received pay-offs respectively converted into Indian rupees and into euros).

The implications of the three aforementioned Justice Views in the context of transcontinental game between Indian and French are straightforward and contrasted. The Formal Equity view would maintain that Indian and French receive the same amount of dollars. The Compensation View would recommend that Indian students receive more in the game as, overall, they are worst-off than French students. Finally, since the actual exchange rates and the prices structures are such that, for a given amount of money, Indian students can buy more than French students, the Local Equity view would advocate that French should receive more in the bargaining.

It is well known that Justice norms are relevant for describing individual behavior in bargaining situations in general and ultimatum games in particular. Norms provide reference points to the individuals, who can either directly implement the norm or anchor their strategic reasoning to a common reference. This allows raising the main question of this paper: Which (if any) notion of Equity is relevant in order to describe bargaining behavior? 


\section{The Ultimatum game: standard results and explanations.}

To test the various views of practical justice, we implemented an Ultimatum Game (henceforth UG). In the UG two individuals have to reach an agreement about how to divide a good that is valuable to both them: a sum of money, a stake, a pie. In the sequential form of the UG, the first player (the sender) makes an offer about the division of the pie to the second player (the receiver). If the receiver accepts the offer, she receives the offered amount while the sender's payoff is the stake minus the offer. If the receiver rejects the offer, both players obtain a zero payoff.

Under the assumptions that players are rational, risk-neutral and have perfect and common knowledge, standard theory predicts that the receiver accepts any offer made by the sender. Any division of the stake is sustained by a Nash equilibrium, even the one where the sender makes an offer of zero to the receiver (and the latter accepts). In the sequential version of the $\mathrm{UG}$, however, there is a unique sub-game-perfect equilibrium: the dominant strategy for the receiver is to accept any offer, even the smaller one say epsilon. In the larger sub-game (which, in the UG, coincides with the game it-self) the strategy of the sender is to offer epsilon.

UG has been the object of an extensive experimental work since the first experiment reported by Güth et al., (1992). This is for at least two reasons: the simplicity of the game and the large empirical puzzling evidence on it. The most striking observations are the following: offers that are inferior to the $20 \%$ of the stake are rejected with a probability that exceeds onehalf, and the average offer is between 30 and $40 \%$ of the stake, depending on how high the probability of rejection is anticipated by the senders ${ }^{6}$.

The discrepancy between theory and empirical evidence has proved to be very robust to the experimental protocol retained (where factors like context, subjects, kind and size of the stake, numbers of rounds, etc. have been variously specified; see Camerer, 2003, for an extensive discussion). In particular, UG was experimented in different countries ${ }^{7}$ (since Roth

\footnotetext{
${ }^{6}$ For surveys on the UG the reader is referred to Güth (1995), Roth (1995),or Camerer (2003).

${ }^{7} \mathrm{We}$ are not aware of Indian studies or between countries ultimatum games.
} 
et al., 1991, and Buchan et al., 1997). Although observations may differ from one country to another, the main qualitative findings are robust, as showed by Oosterbeek et al (2004) ${ }^{8}$.

Several explanations have been provided to reconcile the apparent inconsistency of standard theory and empirical evidence. Most of them paid attention to the social norms that individuals would bring into the game and that would affect their behavior beyond what standard theory commonly assumes. According to these explanations, the utility that players may derive from the game would incorporate social considerations such as the relative standing of each player after the bargaining is concluded, and the way the agreement is reached. On one hand, a large set of models has focused on the feelings of envy or of injustice that very unequal bargaining outcomes trigger. The degree of envy or aversion to inequality determines to what extent a division of the stake will be accepted when it is different from the equal split (Bolton 1991, Fehr and Schmidt 1999). On the other hand, models of intentional or reciprocal behavior (Rabin 1993, Dufwenberg and Firchsteiger 1998) assume that a relevant rationale for action is to reciprocate what one's opponent is expected to do or to reciprocate what she actually does. Models of intentional or reciprocal behavior also incorporate notions of fairness or justice, not directly as an argument of the individual's utility function, but mediated by the individual's understanding of what is the norm in a given circumstance. Fairness is a rewarding response to fairness as well as unfairness is a retaliating response to selfishness; in the UG, a receiver accepts an offer only when she considers it is sufficiently fair and rejects it otherwise.

\section{Interpersonal comparisons and the transcontinental design}

In most experiments the assumption that the marginal value of money is the same for all players is reasonable, since subjects are anonymously drawn from the same population; hence ex-ante inequality (or other "inborn" difference) cannot be taken into consideration. ${ }^{9}$ On the

\footnotetext{
${ }^{8}$ The most remarkable exceptions are the UG experiments run in 17 small-scale societies by Heinrich et al. (2001). Overall, offers varied substantially among these societies and rejections' behaviors were less homogenous than usual. However, no clear pattern emerged: in some societies rejections barely occurred -even at very low offers- while in some others respondents behaved very toughly, rejecting even equal split. As a plausible explanation of such variability, authors put forward the diversity of social institutions and fairness norms across these societies. These studies are not directly relevant for us, since France and India are large-scale societies in which money is the usual mean of exchange.

${ }^{9}$ In the standard version of the UG the amount to be divided is equal for both players, thus "a token is a token" for both the sender and the receiver. If it is common knowledge that (a) the monetary value of the token is the same for both players and (b) the utility of money is the same for both players, it is correct to assume that the
} 
other hand, two players that are not ex-ante equally wealthy may give a different marginal value to the money earned in the negotiation.

Although they do not relax the assumption of identical marginal value of money, Kagel et al. (Kagel et al. 1996) discuss how comparison of utilities can affect individual behavior when players negotiate over tokens with different monetary values. They can test whether the relevant rule for action is the willingness to compensate for different final utilities (expressed in real pay-offs). Two different exchange rates are used to convert experiment token payoffs in actual money payoffs, higher exchange rate for the sender or for the receiver. ${ }^{10}$ Experimental evidence suggests that when senders had higher exchange rate, offers were at about the formally equal split during the first three rounds of the game and increased afterward as rejection rates were very high $(53 \%)$. When receivers had higher conversion rates, senders' offers were not materially different from the equal money split offer ( 25 out of 100). On average, rejection rates were $14 \%$. Hence, formal equality does not appear as the unique and absolute sharing norm.

The main innovation in our experimental design is the following. We let it be common knowledge that players participating in the ultimatum game were different ex-ante in two respects. First, they most likely had a different monetary value of the experimental currency (US dollar) because with one dollar one can buy much more goods in India than in France (about four or five times more). In a certain way, for this part of the design, we replicate the protocol of Kagel et al.. Second, they also probably had a different overall income because the per capita GDP is much larger in France than in India (about 50 times larger when the sessions of the experiment were conducted). With respect to Kagel et al.'s study, this second element is original. Our goal is to study how this twofold source of diversity between players (the game-related one and the actual life-circumstances one) affects the comparison of utility that players perform during the bargaining. The design of the experiment consists of "transcontinental" treatments (sender and receiver from different countries) and of "national" or "within country" treatments (sender and receiver from the same country) as a benchmark.

marginal value of one token left for the receiver is equal to the marginal value of the token the sender renounces to.

${ }^{10}$ If fairness is the relevant rationale for action in this design, the following results should be observed: when the exchange rate is higher for the sender, he should offer more than the equal split in order to grant both herself and her opponent with the same amount of money. By contrast, when the receiver benefits from a hight exchange rate, the sender should offer less than the equal split. In both cases, it is assumed that the division of the stake is the mean by which final utility of money equality is achieved. 
As explained above, interpersonal comparison between players may in principle occur at different levels and thus variously influence their behavior in the game. Our results clearly point out that the relevant reference point for such comparison is the equality in real terms of game-related pay-offs, which is consistent with the Local benefits theory of distributive justice and inconsistent with both the Compensation and the Formal equity theories.

The plan of the paper is the following: Section 2 describes the experimental protocol and the results. Section 3 is devoted to the theory: we develop four variants of the linear Aversion to Inequity model (Fehr and Schmidt, 1999) that allow distinguishing between local and global notions of inequity expressed in real or nominal terms. On the basis of these models, we can submit the conclusion that the relevant notion of Inequity is the local one expressed in real terms. Section 4 briefly discusses the relevance of this point with respect to our understanding of which kinds of justice norms are internalized by the individuals.

\section{The experiment}

\section{The experimental protocol ${ }^{11}$}

The design of the experiment consists of four treatments. Two treatments are transcontinental: FtoI (a French sender makes an offer to an Indian receiver) and ItoF (an Indian sender makes an offer to a French receiver). And two treatments are within-country benchmark treatments: ItoI (an Indian sender makes an offer to an Indian receiver) and FtoF (a French sender makes an offer to a French receiver).

\begin{tabular}{|r|c|c|c|c|} 
Type of treatment & Transcontinental & Transcontinental & Within-country & Within-country \\
\hline Treatment & $\begin{array}{c}\text { Sender : Indian } \\
\text { Receiver : French }\end{array}$ & $\begin{array}{c}\text { Sender : French } \\
\text { Receiver : Indian }\end{array}$ & $\begin{array}{c}\text { Sender : Indian } \\
\text { Receiver : Indian }\end{array}$ & $\begin{array}{c}\text { Sender : French } \\
\text { Receiver : French }\end{array}$ \\
\hline Code of treatment & ItoF & FtoI & ItoI & FtoF \\
\hline
\end{tabular}

Table 1. Experimental Treatments

In both transcontinental and within-country experiments, twenty subjects participated in each session and played six one-shot Ultimatum Games with the "absolute stranger" protocol. In

\footnotetext{
${ }^{11}$ The reader is referred to the appendix for the English version of the instructions distributed to the subjects.
} 
each game, the amount to be divided was 10 US dollars, offers could be made in halves of dollar and two games out of six were paid. The conversion rate used for the payment was the current exchange rate of the US dollar into the local currency (Euro and Indian Rupee) ${ }^{12}$. The exchange rates used were common knowledge. Moreover, the subjects received a sum of 2 US dollars for showing up at the experiment.

For the transcontinental treatments, the subjects were indicated that they were to play with Indian (French) students, and that the game decisions would have been transmitted via an Internet-Chat Connection. Some basic pieces of information were provided to subjects: the per capita GDP of India and France. In addition, the instructions specified the purchasing power of one dollar in each country, by indicating the price in US dollars of selected commodities in both countries. For this purpose, we selected items that were likely to be part of students' expenditures in both countries: coffee in the university campus, cinema ticket, music CD etc. ${ }^{13}$

The experiment was run with a paper and pencil protocol. To make easier the logistics of the experiment, a single treatment is apply for each session. In transcontinental sessions experimentalist in each country transmitted decisions through an Internet-Chat Connection. In each country, the subjects were gathered in a classroom and received the instructions and the experimental material (game-cards, ID, envelopes). After the instructions were read and a test of understanding had been conducted, the experiment was started. In the senders' classroom, the subjects were asked to write down their offer and to put the offer in the envelope. Once all the subjects had finished, the envelopes were collected and transmitted by the experimentalist to the other country. Offers were then copied in the receivers' cards and distributed to the subjects; the receivers were then asked to take their decision to accept or to reject the offer. The receivers' cards were then collected, and acceptances and refusals were transmitted to the senders in the other country. For the within-country experiment the procedure was roughly the same with the exception of decisions transmission. In the latter treatments, in fact, senders and receivers sat in two different rooms and communication of subjects' decision was carried out in a third room by experimentalists. The procedure was repeated six times. Then the random

\footnotetext{
12 The exchange rate used for Euro was $\$ 1$ for $€ 1.1$. The exchange rate used for Indian Rupee was $\$ 1$ for 47 Rps.

For a complete presentation of purchasing power information used in the experiment, the reader is referred to the instructions in the appendix.
} 
drawing was done and the two selected rounds for the final payment were communicated to subjects.

French students received instructions in French and Indian students received instructions in English. A special attention was paid to the instructions' translation: the instructions were firstly drafted in English following standard UG instructions; they were then translated into French. The final version of instructions in both languages was done after a common revision, in order to make instructions equally understandable for all the subjects.

\section{Main predictions}

Taking into account the characteristics of the Transcontinental Protocol (difference of beyond-game status, difference of purchasing power, equal nominal value of the stake), one may expect three kinds of behavior.

If the nominal value of the stake matters, then no different behavior should be observed in transcontinental treatments with respect to previous within country experiments, nor one should find any significant differences between FtoI and ItoF treatments. The modal observation should be the equal nominal split.

If the first factor (beyond-game wealth effect) is relevant, we should observe that the outcomes of bargaining are always in favor of Indian subjects (the interaction would allow for compensation between ex ante differences between players).

Finally, if the second factor plays a crucial role (different value of one dollar in the two countries), then we should observe unequal splits occurring in the opposite direction, that is in favor of French subjects. The rationale behind this peculiar division is to equalize the real pay-off of players.

As specified above, our hypothesis is that the country of residence of players may play a role in shaping the interpersonal comparison of utility. Specifically, we do not assume that cultural norms themselves exert such an influence but, instead, that the country of residence of the players indirectly affects the equity norm that sustains the agreement: the equity norm is endogenously settled as to account for the differences of purchasing power and of income between the two countries. In principle, however, we cannot rule out the fact that Indian subjects and French subjects behave differently in ultimatum games for reasons intrinsically related to their culture. It is thus necessary to provide a counter-proving test, which 
invalidates the cultural discrimination story. The comparison between within-country treatments is the natural test for this latter point.

No standard experimental test of the Ultimatum Game has previously been conducted in India or France (to our knowledge), so we needed benchmarks cases to be compared with transcontinental treatments. The objective of running with-in country treatments is twofold. First, it allows establishing if bargaining behavior is the same than that previously observed using an almost identical protocol and in countries like United States, Israel, Japan etc. In particular, we want to see if there are any differences between French to French negotiation and Indian to Indian one. Secondly and more importantly, we want to compare the within country and the transcontinental treatments to test whether the identity of the subjects affect bargaining outcomes. The within-India and within-France treatments were conducted with the same nominal stake of $\$ 10$. Note that, in real terms, the stake is thus higher in the Indian treatment.

\section{Results}

All in all, we ran eleven sessions during 2002 and 2003. Six transcontinental sessions between Delhi and Grenoble were organized: three ItoF sessions, and three FtoI sessions. Moreover, three ItoI sessions were run in Delhi and two FtoF sessions were run in Grenoble. ${ }^{14}$ On the whole, the results of the FtoF sessions are consistent with the usual results of standard ultimatum game experiments. That is why we only ran two sessions with this treatment. The date and the average earning of each session are reported in Table 2. On average, withincountry sessions lasted one hour, while transcontinental sessions lasted one hour and half.

\footnotetext{
${ }^{14}$ A pilot session was run for the transcontinental protocol of the UG. This allowed improving some aspects of the experimental procedure and checking the feasibility and the effectiveness of the experimental communications between countries.
} 


\begin{tabular}{|c|c|c|c|c|c|}
\hline $\begin{array}{l}\text { Session } \\
\text { ID }\end{array}$ & Date & Treatment & $\begin{array}{l}\text { Number } \\
\text { of } \\
\text { subjects }\end{array}$ & $\begin{array}{c}\text { Average } \\
\text { Earning in US\$ }\end{array}$ & $\begin{array}{c}\text { Average Earning in } \\
\text { Local Currency } \\
\text { (Rps: Indian Rupees, } € \\
\text { : Euro) }\end{array}$ \\
\hline ItoF-S1 & $02 / 19 / 2002$ & $\begin{array}{l}\text { Indian Senders- } \\
\text { French Receivers }\end{array}$ & $\begin{array}{c}10 \\
\text { couples }\end{array}$ & $\begin{array}{l}\text { Senders: } \$ 10.75 \\
\text { Receivers: } \$ 7.42\end{array}$ & $\begin{array}{c}\text { Senders: } 515 \text { Rps } \\
\text { Receivers: } € 8.1\end{array}$ \\
\hline ItoF-S2 & $02 / 21 / 2002$ & $\begin{array}{l}\text { Indian Senders- } \\
\text { French Receivers }\end{array}$ & $\begin{array}{c}10 \\
\text { couples }\end{array}$ & $\begin{array}{c}\text { Senders: } \$ 8.77 \\
\text { Receivers: } \$ 8.15\end{array}$ & $\begin{array}{c}\text { Senders: } 420 \text { Rps } \\
\text { Receivers: } € 9\end{array}$ \\
\hline ItoF-S3 & $02 / 11 / 2003$ & $\begin{array}{l}\text { Indian Senders- } \\
\text { French Receivers }\end{array}$ & $\begin{array}{c}10 \\
\text { couples }\end{array}$ & $\begin{array}{l}\text { Senders: } \$ 10.75 \\
\text { Receivers: } \$ 8.27\end{array}$ & $\begin{array}{c}\text { Senders: } 515 \text { Rps } \\
\text { Receivers: } € 9.1\end{array}$ \\
\hline FtoI-S1 & $02 / 20 / 2002$ & $\begin{array}{l}\text { French Senders- } \\
\text { Indian Receivers }\end{array}$ & $\begin{array}{c}10 \\
\text { couples }\end{array}$ & $\begin{array}{l}\text { Senders: } \$ 14.45 \\
\text { Receivers: } \$ 6.85\end{array}$ & $\begin{array}{c}\text { Senders: } € 15.9 \\
\text { Receivers: } 330 \mathrm{Rps}\end{array}$ \\
\hline FtoI-S2 & $02 / 22 / 2002$ & $\begin{array}{l}\text { French Senders- } \\
\text { Indian Receivers }\end{array}$ & $\begin{array}{c}10 \\
\text { couples }\end{array}$ & $\begin{array}{l}\text { Senders: } \$ 17.35 \\
\text { Receivers: } \$ 6.45\end{array}$ & $\begin{array}{c}\text { Senders: } € 19.1 \\
\text { Receivers: } 310 \text { Rps }\end{array}$ \\
\hline FtoI-S3 & $02 / 12 / 2003$ & $\begin{array}{l}\text { French Senders- } \\
\text { Indian Receivers }\end{array}$ & $\begin{array}{c}10 \\
\text { couples }\end{array}$ & $\begin{array}{l}\text { Senders: } \$ 14.8 \\
\text { Receivers: } \$ 7.9\end{array}$ & $\begin{array}{c}\text { Senders: } € 16.3 \\
\text { Receivers: } 380 \text { Rps }\end{array}$ \\
\hline ItoI-S1 & $02 / 18 / 2002$ & $\begin{array}{l}\text { Indian Senders- } \\
\text { Indian Receivers }\end{array}$ & $\begin{array}{c}10 \\
\text { couples }\end{array}$ & $\begin{array}{l}\text { Senders: } \$ 14.4 \\
\text { Receivers: } \$ 6.6\end{array}$ & $\begin{array}{l}\text { Senders: } 682 \mathrm{Rps} \\
\text { Receivers: } 321 \mathrm{Rps}\end{array}$ \\
\hline ItoI-S2 & $02 / 03 / 2003$ & $\begin{array}{l}\text { Indian Senders- } \\
\text { Indian Receivers }\end{array}$ & $\begin{array}{c}10 \\
\text { couples }\end{array}$ & $\begin{array}{l}\text { Senders: } \$ 13.2 \\
\text { Receivers: } \$ 7.6\end{array}$ & $\begin{array}{l}\text { Senders: } 634 \text { Rps } \\
\text { Receivers: } 365 \text { Rps }\end{array}$ \\
\hline ItoI-S3 & $02 / 03 / 2003$ & $\begin{array}{l}\text { Indian Senders- } \\
\text { Indian Receivers }\end{array}$ & $\begin{array}{c}10 \\
\text { couples }\end{array}$ & $\begin{array}{l}\text { Senders: } \$ 11.2 \\
\text { Receivers: } \$ 7.7\end{array}$ & $\begin{array}{c}\text { Senders: } 538 \text { Rps } \\
\text { Receivers: } 370 \text { Rps }\end{array}$ \\
\hline FtoF-S1 & $10 / 23 / 2002$ & $\begin{array}{l}\text { French Senders- } \\
\text { French Receivers }\end{array}$ & $\begin{array}{c}10 \\
\text { couples }\end{array}$ & $\begin{array}{l}\text { Senders: } \$ 13.1 \\
\text { Receivers: } \$ 9.1\end{array}$ & $\begin{array}{c}\text { Senders: } € 14.3 \\
\text { Receivers: } € 10.1\end{array}$ \\
\hline FtoF-S2 & $10 / 24 / 2002$ & $\begin{array}{l}\text { French Senders- } \\
\text { French Receivers }\end{array}$ & $\begin{array}{c}11 \\
\text { couples }\end{array}$ & $\begin{array}{l}\text { Senders: } \$ 13.1 \\
\text { Receivers: } \$ 9.1\end{array}$ & $\begin{array}{c}\text { Senders: } € 14.3 \\
\text { Receivers: } € 10.1\end{array}$ \\
\hline
\end{tabular}

Table 2. Sessions details

As already explained, every subject played consecutively six ultimatums, changing opponent at each new round. For each session, the main statistics by treatment for all periods polled and for the last period are reported in Table 3. 


\begin{tabular}{|r||r||r||r||r|}
\hline Sessions & FtoF $^{15}$ & ItoF & ItoI & FtoI \\
\hline \hline $\begin{array}{r}\text { Number of } \\
\text { observations }\end{array}$ & 120 & 180 & 180 & 180 \\
$(20)$ & $(30)$ & $(30)$ & $(30)$ \\
\hline \hline Average Offer & 3.48 & 3.92 & 3.53 & 2.63 \\
& $(3.70)$ & $(3.87)$ & $(2.98)$ & $(2.37)$ \\
\hline \hline $\begin{array}{r}\text { Standard } \\
\text { Deviation }\end{array}$ & 1.14 & 1.08 & 1.46 & 1.01 \\
$(0.68)$ & $(1.06)$ & $(1.52)$ & $(0.98)$ \\
\hline \hline $\begin{array}{r}\text { Global Frequency } \\
\text { of Rejection }\end{array}$ & $\begin{array}{r}22.5 \% \\
(0.1 \%)\end{array}$ & $20.0 \%$ & $9.4 \%$ & $12.2 \%$ \\
$(0.07 \%)$ & $(0.13 \%)$ & $(0 \%)$ \\
\hline \hline $\begin{array}{r}\text { Average Offer } \\
\text { Rejected }\end{array}$ & 2.4 & 2.9 & 2.1 & 1.7 \\
\hline
\end{tabular}

Table 3. Global statistics all periods confounded (last period)

Overall, the results of ItoF and FtoF treatment sessions are in line with what is commonly observed in standard UG experiment. In fact, our results do support previous findings such that any offer below the $20 \%$ of the stake is rejected with a probability of $(0,4-0,6)$ and that the average offers are between 30 and $40 \%$ of the stake. By contrast, what we observe under the treatment FtoI, but also under the treatment ItoI for the last periods, is radically different from the usually observed facts. Mean offers are low and, conditionally to these offers, rejection rates are significantly lower than what is commonly observed in standard UG.

\section{Offers and rejection decisions.}

Figure 1 reports the distribution of the offers and the rate of rejection. The subjects had the possibility of making offers with halves of dollars, but most of the offers were integers; therefore, in order to have more readable pictures, we pool the offers $\$ 0.5$ with $\$ 1, \$ 1.5$ with $\$ 2$, etc. Offers of $\$ 0$ have not been observed and offers higher than $\$ 5$ are very rare. $^{16}$

\footnotetext{
${ }^{15}$ Eleven couples participated in one of these two sessions. In spite of going successfully through both instructions and test of understanding, one subject misunderstood the protocol and sent to her opponent an offer containing the amount of money he intended to keep for himself. This happened for all the duration of the experiment. He sent proposals of $\$ 6$ and $\$ 7$. After having analysed the whole results, we decided to exclude observations concerning him (and related responses by his opponents), but we considered valuable the rest of the data. We could in fact verify that the misunderstanding of the subject did not sensibly affect the behaviour of players who met him when they played with the others senders

${ }^{16}$ Offers higher than $\$ 5$ were observed with the following frequency : two observations out of 120 for the FtoF treatment, two observations over out of 180 for FtoI, 3 observations out of 180 for ItoF and 3 out of 180 for ItoI.
} 

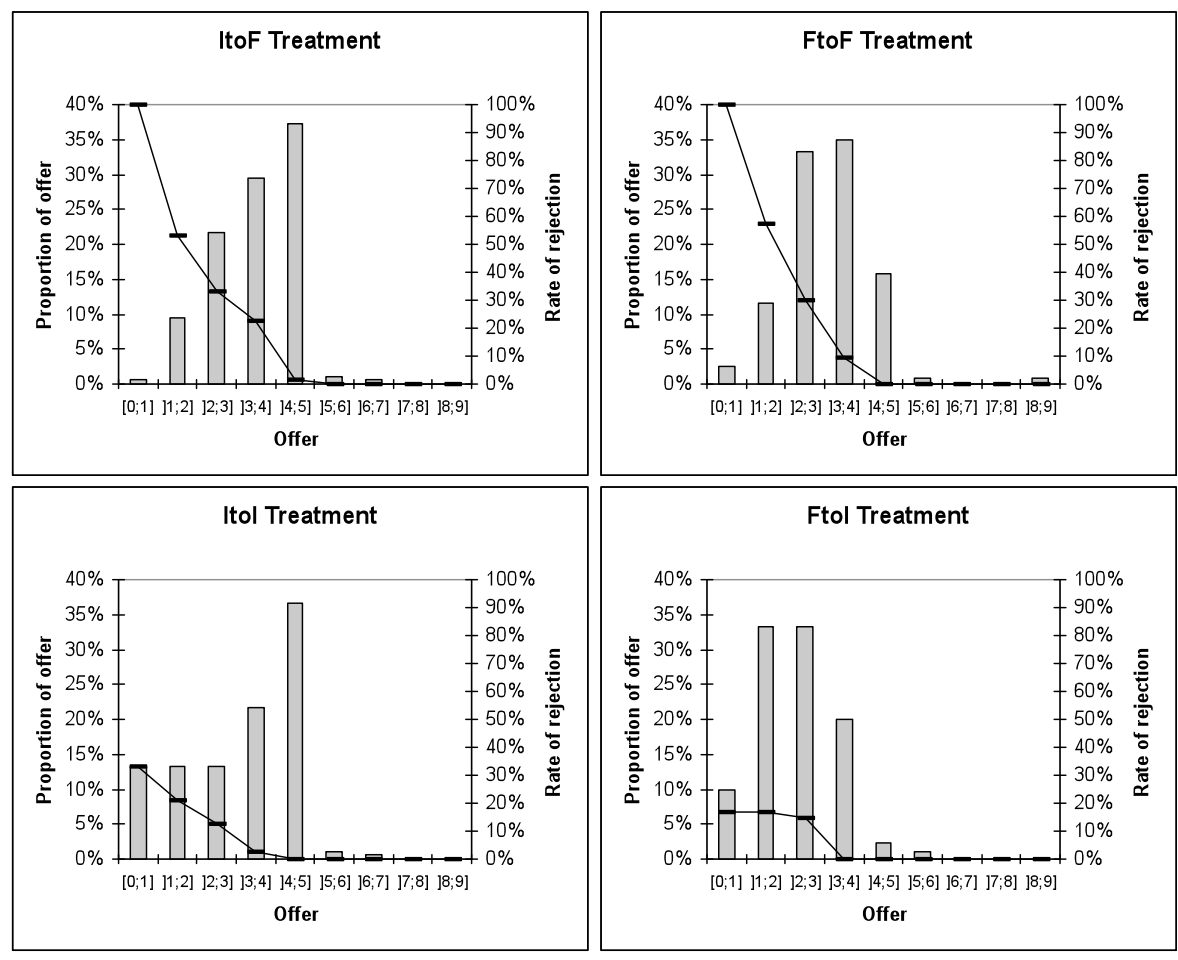

Figure 1. Offers distribution and rate of rejection by amount offered

(The columns represent the percentage of offers and the curve represents the rate of rejection)

The comparison of offers between ItoF and FtoI treatments is clear: the Indian senders were more generous towards French receivers than French senders towards Indian receivers. The average offer in the ItoF treatment is $\$ 3.92$, while it is only $\$ 2.63$ in the FtoI interactions. At the same time, Indian receivers were more conciliating than the French receivers. In fact rejection rates for offer below $\$ 3$ is $19 \%$ in the FtoI treatment. The corresponding feature for the ItoF treatment is $55 \%$. The difference between these rates can be ascribed to a different rejection threshold between Indian and French subjects. The different thresholds across Indian and French students are also confirmed by within-country treatments, where senders met receivers of same nationality. In FtoF treatment, the rejection rate relative to offers less than $\$ 3$ is $60 \%$ while it is $25 \%$ in the ItoI treatment.

From these results we conclude that the rejection threshold of Indian subjects is definitely lower than the French subjects' one and that the difference between the minimum amount of money that a player is ready to accept is a relevant factor for understanding the outcome of 
interactions. In addition, our data show that the rejection threshold is not common knowledge among players, even when one plays against someone coming from her same country. The evolution of proposition along the six rounds of the game can be explained by the fact that senders seek the acceptation threshold until they find the 'right' one.

\section{Dynamics pattern}

The round mean offer and the round rate of rejection for the various treatments are reported in Figure 2.
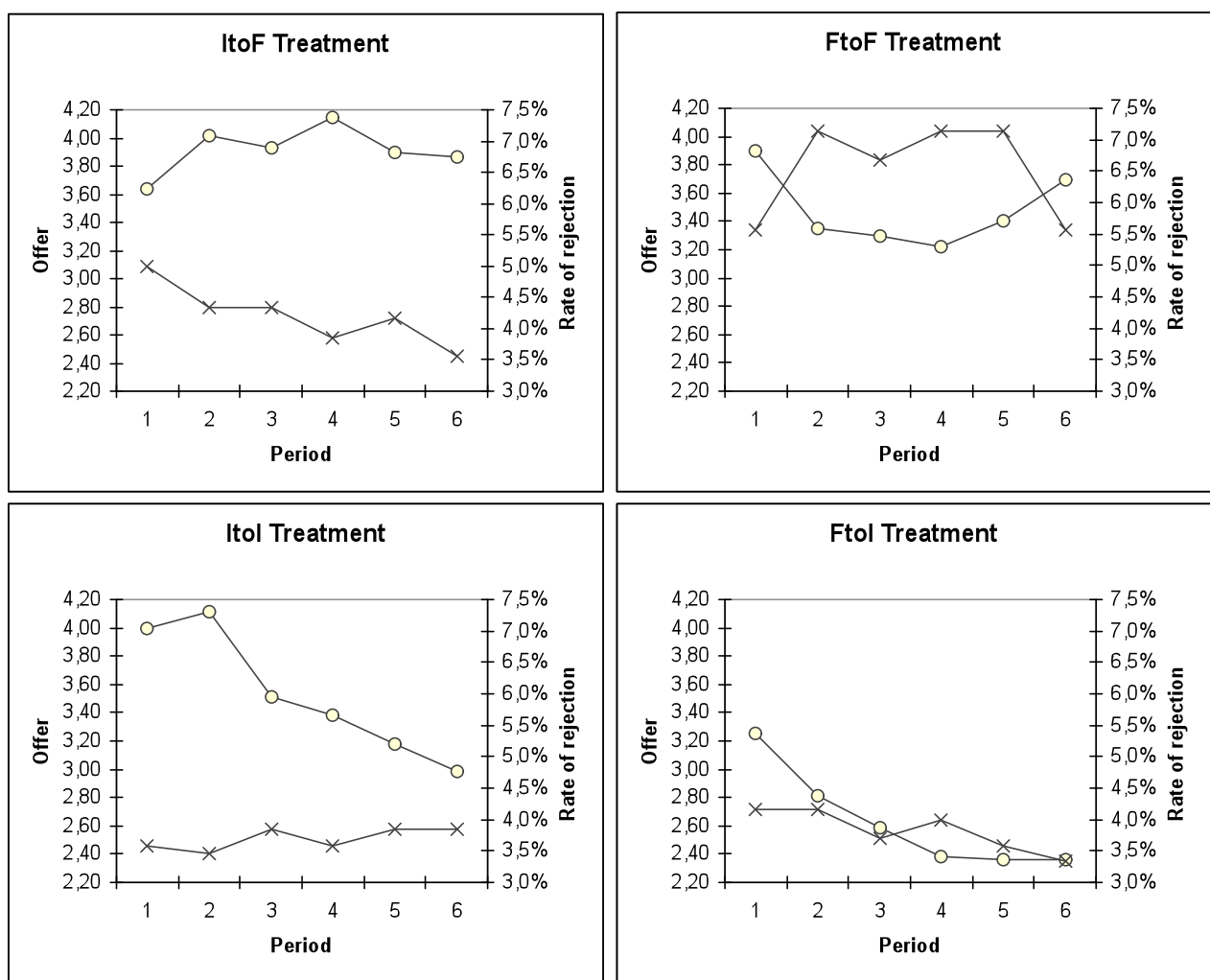

Figure 2. Offer and rate of rejection evolution

(The circle curve represents the average offer and the cross curve represents the rate of rejection)

To start with, it is instructive to see how the first round offer is very similar across all four treatments. The mean offer is around $\$ 3.3$ in the FtoI treatment and nearly $\$ 4$ in the ItoI treatment. Excepted for these two treatments, the comparison between mean offers by 
treatment does not reveal any statistically significant difference (at the $5 \%$ threshold ${ }^{17}$ ). With the only exception of FtoI treatment, the observed offers are not different from what is usually found in this game.

By contrast, we found that, when repeating the game, two different trends emerged depending on the receiver's nationality, and irrespective of the sender's nationality. In particular, offers made to Indian receivers decreased progressively over successive rounds while offers sent to French receivers almost remain unchanged from the first period. The pattern of offers over time can be explained by both the receivers' propensity to reject an offer and by the consequences of rejection and acceptation on senders' following strategy. Concerning the latter, the reaction of senders seems to be quite homogenous (see Table 4).

\begin{tabular}{|r||c|c|c|c||c||c|}
\cline { 2 - 7 } \multicolumn{1}{|c|}{} & \multicolumn{2}{c|}{ Offer rejected in the previous period } & \multicolumn{2}{c|}{ Offer accepted in the previous period } \\
\hline Treatment & $\begin{array}{c}\text { Increase } \\
\text { offer }\end{array}$ & $\begin{array}{c}\text { Maintain } \\
\text { offer }\end{array}$ & $\begin{array}{c}\text { Decrease } \\
\text { offer }\end{array}$ & $\begin{array}{c}\text { Increase } \\
\text { offer }\end{array}$ & $\begin{array}{c}\text { Maintain } \\
\text { offer }\end{array}$ & $\begin{array}{c}\text { Decrease } \\
\text { offer }\end{array}$ \\
\hline \hline FtoF & $17(68 \%)$ & $8(32 \%)$ & 0 & $3(4 \%)$ & $49(65 \%)$ & $23(31 \%)$ \\
\hline FtoI & $15(68 \%)$ & $7(32 \%)$ & 0 & $4(3 \%)$ & $73(57 \%)$ & $51(40 \%)$ \\
\hline ItoF & $29(85 \%)$ & $5(15 \%)$ & 0 & $7(6 \%)$ & $73(63 \%)$ & $36(31 \%)$ \\
\hline ItoI & $10(77 \%)$ & $3(23 \%)$ & 0 & $19(14 \%)$ & $59(43 \%)$ & $59(43 \%)$ \\
\hline Total & 142 & 46 & 0 & 66 & 508 & 338 \\
\hline
\end{tabular}

Table 4. Offer evolution after a rejection or an acceptance

In fact, senders increased their offer or kept it unchanged after a refusal, while they maintained it unchanged or diminished it when their proposal was accepted. At the same time, as said above, the Indian acceptation threshold is lower than the French one. Overall, we observe that treatments where receivers are Indian are precisely treatments such that offers fall over time ${ }^{\mathbf{1 8}}$. Senders keep diminishing their offers without triggering any negative reaction on the other side. In the sixth round, the mean offer is 'only' $\$ 2.4$ in the FtoI treatment and $\$ 3$ in the ItoI treatment.

When receivers are French, the story is completely different. In these sessions, refusal occurs more often whenever offers are below $\$ 3$. By way of consequence, senders do not

\footnotetext{
${ }^{17}$ The null hypothesis that offers are identical across treatments ItoI and FtoI is rejected by a Mann-Whitney test at the .05 level $(\mathrm{P}=0.024)$. We can not reject the null hypothesis that offers are identical across the others treatments.
} 
significantly vary their offer over the game and this is why proposed and accepted splits almost remain unchanged during the six rounds. In the last round, the mean offer is $\$ 3.7$ in the FtoF treatment and is $\$ 3.9$ in the ItoF treatment. A statistical test allows us to conclude that no significant difference exists between first round offers and last round offers for these treatments. $^{19}$

From these findings, it appears that the repetition of the game, even with different opponents, allows senders to identify the threshold up to which receivers are ready to accept offers. Senders' learning is made out in terms of offer reduction, which can be seriously sanctioned by refusals when the threshold is overcome. Thus, the last period offers integrate senders' learning about opponents' propensity to accept. For each treatment, the main statistics concerning the last period are reported in Table 5.

\begin{tabular}{||c||c||c||c||c|}
\hline Treatment & ItoF & FtoI & ItoI & FtoF \\
\hline \hline Number of couples & 30 & 30 & 30 & 20 \\
\hline \hline Average Offer & 3.87 & 2.37 & 2.98 & 3.70 \\
\hline \hline $\begin{array}{r}\text { Standard Deviation } \\
\hline \hline \begin{array}{r}\text { Global Frequency } \\
\text { of Rejection }\end{array}\end{array}$ & 1.06 & 0.98 & 1.52 & 0.68 \\
\hline
\end{tabular}

Table 5. Last period statistics

Data reported in Table 5 show that proposals addressed to French respondents are in line with standard stylized facts in UG experiments. For the ItoF treatment, the mean offer is respectively $\$ 3.87$. This observation is similar in the FtoF treatment, where the mean offer is $\$ 3.70$. On the other hand, the last period proposals made to Indian are significantly lower than that. In fact, mean offers for FtoI and ItoI treatments are respectively \$2.37 and \$2.98.

It emerges from the statistical analysis that senders make different offers according to the nationality of responders. In fact French senders' proposals are significantly different when

\footnotetext{
${ }^{18}$ The null hypothesis that offers are identical across the first and the last period is rejected by a Wilcoxon matched-pairs signed-ranks test at the .01 significance level for treatments ItoI $(\mathrm{P}=0.0087)$ and treatment FtoI $(\mathrm{P}=0.0013)$

${ }^{19}$ The null hypothesis that offers are identical across the first and the last period is not rejected by Wilcoxon matched-pairs signed-ranks test at the .01 level for treatments ItoF $(\mathrm{P}=0.2993)$ and treatment FtoF $(\mathrm{P}=0.4420)$.
} 
they are to be received by an Indian or a French respondent ${ }^{20}$. Analogously, offers coming from the Indian senders vary with the respondents' nationality ${ }^{21}$.

For a more comprehensive analysis of the offer and rejection decisions, we estimate two panel data models since several decisions are made by the same individuals as sender or receiver. Firstly, we estimate a random-effects panel data model to identify the determinants of the amount the sender offers to the receiver. The independent variables include the treatments with the FtoF treatment as the reference and a time trend. Table 6 displays the results of these estimations for pooled data.

\begin{tabular}{|c|c|}
\hline & Dependent variable: Offer \\
\hline Constant & $\begin{array}{c}3.849167 * * * \\
(.2061954)\end{array}$ \\
\hline FtoF treatment & Ref. \\
\hline FtoI treatment & $\begin{array}{c}-.8513889 * * * \\
(.2498054)\end{array}$ \\
\hline ItoF treatment & $\begin{array}{c}.4375 * \\
(.2498054)\end{array}$ \\
\hline ItoI treatment & $\begin{array}{c}.0513889 \\
(.2498054)\end{array}$ \\
\hline Period & $\begin{array}{c}-.1057143 * * * \\
(.0203538)\end{array}$ \\
\hline$N$ & 660 \\
\hline$R$-sq. overall & 0.1649 \\
\hline Wald $\chi^{2}$ & 62.35 \\
\hline Prob $>\chi^{2}$ & 0.0000 \\
\hline
\end{tabular}

Table 6 confirms that the offer for the receiver is lower for the FtoI treatment and higher for the ItoF treatment, compared with the baseline treatment (i.e. FtoF treatment). In this way French senders propose a reduced offer when receivers are Indian. On the contrary, Indian

${ }^{20}$ The null hypothesis that offers at the last period are identical across treatments FtoI and FtoF is rejected by a Mann-Whitney test at the .05 level $(\mathrm{W}=302.5$ and p.c. $=0.0000)$.

${ }^{21}$ The null hypothesis that offers at the last period are identical across treatments ItoF and ItoI is rejected by a Mann-Whitney test at the .05 level $(\mathrm{W}=766.0$ and p.c. $=0.0256)$. 
senders offer more money to French receiver than French senders. We do not observe any significant difference between ItoI treatment and FtoF treatment. The time trend is also significant, suggesting that senders decrease their offer as periods are repeated. This result should be explained by the fact that, as shown below, receivers' propensity to reject unfair offers decreases with period repetition.

Secondly, we estimate a random-effects probit model to identify the determinants of the receveirs' decision of acceptation, here again several decisions are made by the same individuals. The independent variables are the amount proposed to the receiver, the treatments, with the FtoF treatment as the reference, and a time trend. Table 7 displays the results of these estimations for pooled data.

\begin{tabular}{|c|c|}
\hline & $\begin{array}{l}\text { Dependent variable: } \\
\text { Responder decision of acceptation }\end{array}$ \\
\hline Constant & $\begin{array}{c}-2.810967 * * * \\
(.5148294)\end{array}$ \\
\hline FtoF treatment & Ref. \\
\hline FtoI treatment & $\begin{array}{c}1.243329 * * * \\
(.3840941)\end{array}$ \\
\hline ItoF treatment & $\begin{array}{l}-.2507209 \\
(.3702512)\end{array}$ \\
\hline ItoI treatment & $\begin{array}{c}1.04595 * * * \\
(.4005683)\end{array}$ \\
\hline Offer for receiver & $\begin{array}{c}.9937613 * * * \\
(.12414)\end{array}$ \\
\hline Period & $\begin{array}{l}.2181058 * * * \\
(.0537193)\end{array}$ \\
\hline$N$ & 660 \\
\hline Log likelihood & -201.54314 \\
\hline Wald $\chi^{2}$ & 67.39 \\
\hline Prob $>\chi^{2}$ & 0.0000 \\
\hline
\end{tabular}

As one can expect, Table 7 shows that the frequency of acceptation increases with the amount offered to receivers. The time trend is also significant: the receiver propensity to 
accept increases as periods are repeated. It is possible to explain this time trend considering that receivers could be more reluctant to reject unfair offer as period are repeated and as they already reject previous offers. Compared to the FtoF treatment, FtoI and ItoI treatments have significant and positive impact on the frequency of acceptation. The impact of the ItoF treatment is not significant. These results suggests that Indian receivers are more willing to accept offers, even unfair, that French receivers, whatever the sender is Indian or French.

To sum up, offers made to Indian subjects are, everything else being equal, more unfavourable than offers made to French subjects. Facing a French sender, an Indian respondent is confronted with lower offer than a French respondent. Similarly, an Indian sender is more likely to offer less when the receiving end of the proposal is an Indian, rather than a French. This finding is at odds with the view of justice as compensation, for which beyond-game wealth differences should matter. Also, our results do not validate the conjecture of equal nominal amounts division. Indeed, the (observed) splits in transcontinental bargaining are significantly different from the (observed) splits in with-in country bargaining. All these facts considered, we shall conclude that the relevant explanation behind TUG is the willingness to compensate for a difference of purchasing power between India and France; to reach this conclusion, a more precise theoretical framework is needed, which is developed in the next section.

\section{Theoretical framework}

\section{Generalities}

Several propositions have been made in the literature to take into account fairness, altruism, status comparisons, and similar phenomenon which may be relevant in our context. ${ }^{22}$

\footnotetext{
${ }^{22}$ Let us consider a bargaining game between player $i$ and player $j$. Models of fairness can be classified as: 1$)$ Model of Altruism (Andreoni and Miller, 2002; Charness and Rabin, 2002) : the utility function of player $i$ is increasing in the payoff of player $j$; 2) Model of Relative income and Envy (Bolton, 1991, Kirchsteiger 1994): the first partial derivative of utility function of player $i$ with respect to the ratio of $i$ 's payoff to $j$ 's payoff is strictly positive when the payoff of player $j$ is inferior to the player $i$ 's one and zero otherwise ; 3) Model of Inequity Aversion (Fehr and Schmidt, 1999 ; Bolton and Ockenfels, 2000): player is altruistic towards other players if their payoff are below an equitable benchmark, but she feels envy when the payoff of the other player
} 
For instance, in a two players game, Fehr and Schmidt (1999) assume that individual $i$ 's preference linearly depends on $i$ 's own payoff and on the difference between this payoff and player $j$ 's (the opponent) one. Their Homo Egalis ${ }^{23}$ maximizes the following utility function $u_{i}$ :

$$
\begin{aligned}
& u_{i}\left(y_{i}, y_{j}\right)=y_{i}-\alpha\left(y_{j}-y_{i}\right) \text { if } y_{j}>y_{i} \\
& \text { and } \\
& u_{i}\left(y_{i}, y_{j}\right)=y_{i}-\beta\left(y_{i}-y_{j}\right) \text { if } y_{j} \leq y_{i}
\end{aligned}
$$

Here $y_{i}$ denotes the payoff of player $i$, and $y_{j}$ denotes the payoff of player $j$. In the base-line model, $y_{i}$ is given by $x_{i}$, the nominal pecuniary payoff of individual $i$. The parameter $\alpha$ captures the equity concern when player $i$ has less than player $j(\alpha>0)$ and $\beta$ captures the equity concern when he has more than his opponent $(0 \leq \beta \leq 1)$. The models that we shall study are four variations of this model, suitable for our context. More precisely we will discuss different specifications of players' payoffs. Firstly, the payoff can be the nominal pecuniary payoff of the individual, or it can be the real payoff of the individual, taking into account the real exchange rate between the goods to be bought in the country of player $i$ and the nominal pecuniary payoff. Secondly, referring to the theories of Justice, when the payoff includes out-of-game wealth, we shall speak of a Global theory whereas a Local theory only involves game payments. We therefore have four variations of the Fehr-Schmidt model. To present the possibilities for the game "payoffs" $y_{i}, y_{j}$, we always denote by $x_{i}$ and $x_{j}$ the monetary earnings of, respectively, player $i$ and player $j$ in nominal dollars.

(1) The ALINom model (Aversion to Local Inequity in Nominal terms): payoffs are game earnings in nominal terms. Then, for player $i$ :

$$
y_{i}=x_{i}
$$

Here we consider that the payoff is the nominal dollar earnings of the individual in this game.

exceeds this level. In the second of these two models, the utility function is assumed to be weakly increasing and concave in player's own payoff; for any given payoff, the utility function is strictly concave in player's $i$ share of total income and obtains a maximum for equal split.

${ }^{23}$ Gintis 2000. 
(2) The ALIReal model (Aversion to Local Inequity in Real terms): payoffs are game earnings in real terms:

$$
y_{i}=\theta_{i} x_{i}
$$

Here we consider that the payoff is the real payoff of the individual, thus $\theta_{i}$. is the real exchange rate between the goods to be bought in the country of player $i$ and the dollar. In our case (and this point was recalled in the instructions) $\theta$ is about four time larger for an Indian subject than for a French subject. We can let:

$$
\theta^{\mathrm{I}}=1 \text { and } \theta^{\mathrm{F}}=.25 \text {. }
$$

(3) The AGINom model (Aversion to Glocal Inequity in Nominal terms): payoffs are aftergame wealth in nominal terms.

$$
y_{i}=R_{i}+x_{i}
$$

Here we consider that the payoff is the nominal wealth of player $i$, including both the game earnings $x_{i}$ and the out-of game wealth $R_{i}$, all expressed in US dollars. In our case (and this point was recalled in the instructions) $R$ is much larger for a French subject than for an Indian subject. and obviously, in both countries $R$ is much larger than the stake of the game (10 dollars):

$$
10<<R^{\mathrm{I}}<<R^{\mathrm{F}} .
$$

(4) The AGIReal model (Aversion to Glocal Inequity in Real terms): payoffs are aftergame wealth in real terms

$$
y_{i}=\theta_{i} R_{i}+\theta_{i} x_{i}
$$

Here we consider that the payoff is the real wealth of player $i$, including both the game earnings $x_{i}$ and the out-of game wealth $R_{i}$. Again, real wealth is much larger for a French subject than for an Indian subject and both are much larger than the stake of the game.

Each of the models above can be solved. Each has a unique sub-game perfect equilibrium. The complete solving of these games is tedious and is done in Appendix 2. It is nevertheless useful to describe the logic at work when solving these games with two figures. For these 
figures, we considerer the ItoF treatment when an Indian makes offers to a French, utility for both players are represented. We suppose that the minimum acceptable amount by French player is $\$ 2$ (which corresponds to a value of $1 / 3$ for $\alpha$ ) and that players payoffs are given by the nominal earnings.

As a function of his nominal share, the utility of a player is always increasing when the share is smaller than the equitable share. This is because increasing the share not only directly increases the player's payoff but also decreases the level of inequality. When the share is larger than the equitable one, the utility is still increasing if the marginal disutility for inequality is not too strong that is when $\beta$ is small as it's represented in Figure 3.

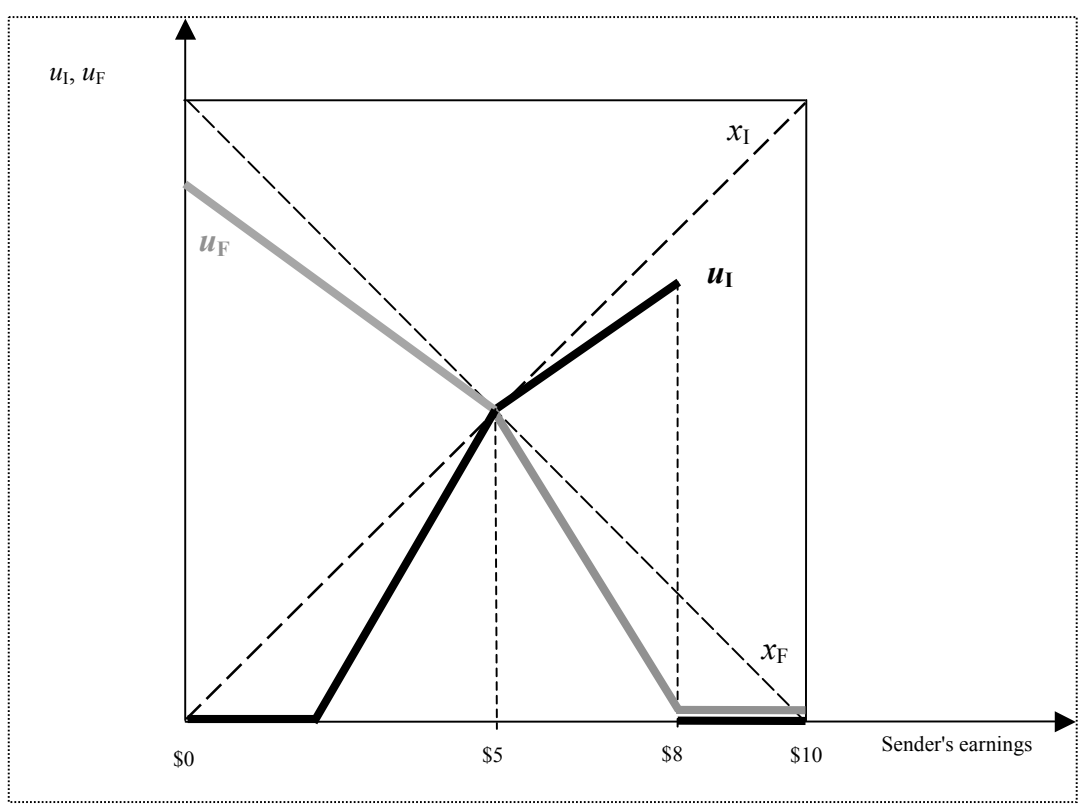

Figure 3. Utility of Indian sender and French receiver with the ALINom model in ItoF treatment and with small $\beta$

In the opposite case, that is large $\beta$, the utility is decreasing because marginal disutility for inequality is not too strong compared to the increase of earnings (Figure 4). 


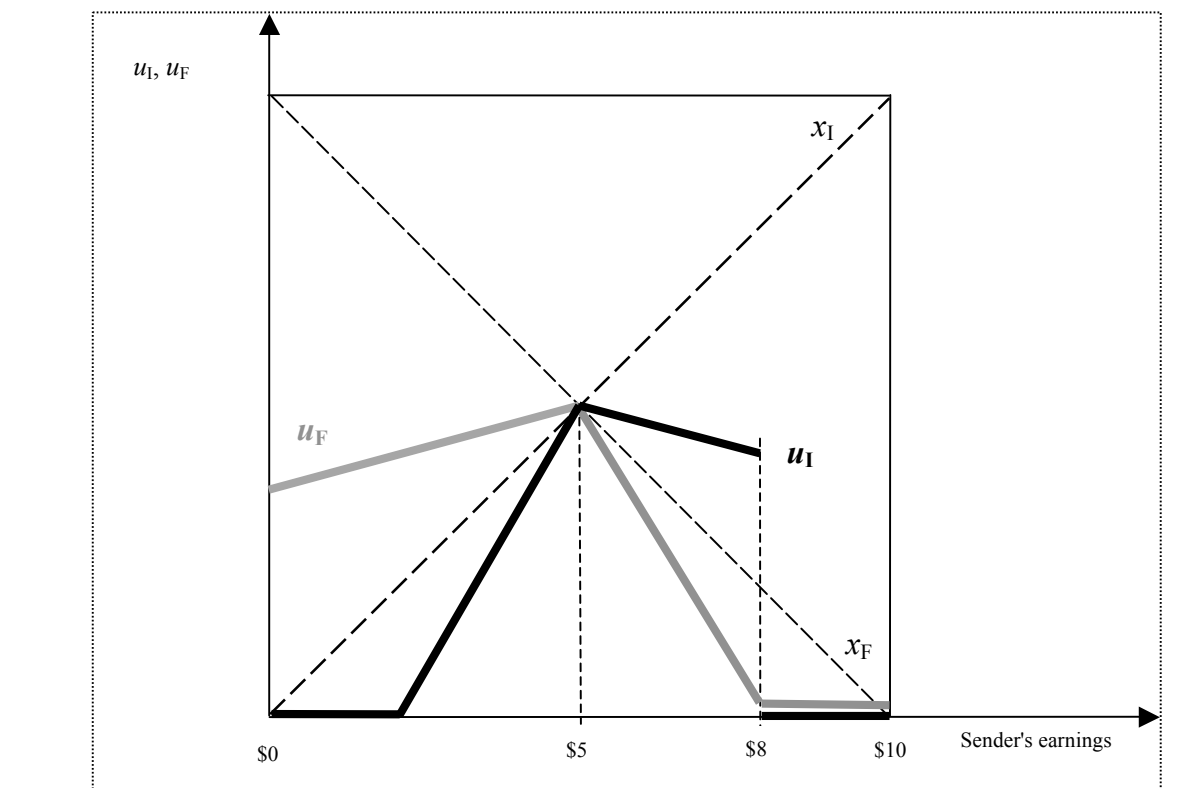

Figure 4. Utitity of Indianr sender and French receiver with the AEfNom model in ItoF- treatment and large $\beta$

It follows that equilibria are of two kinds. For small $\beta$, the equilibrium is always that the sender offers the smallest amount that the receiver prefers to its reservation utility. That is, in Figure 3, the Indian sender should keep $\$ 8$ and offer $\$ 2$ to the French receiver. For larger $\beta$, another kind of equilibrium may appear, in which the equitable share is implemented. Here, as it show in Figure 4, the Indian sender should offer $5 €$ for the French receiver. In a given game, the equilibrium is unique.

\section{Model predictions}

In all what follows, the equilibria are described in terms of the nominal share $\left(x_{j}\right)$ obtained by the receiver.

(1) The ALINom model

The predictions of this model is that outcomes should be the same in all four treatments, a conclusion which is clearly invalidated by the experiment.

(2) The ALIReal models 
The treatments ItoI and FtoF are not distinguished and one has:

ALIReal[FtoI] $<$ ALIReal[ItoI] $=$ ALIReal[FtoF] $<$ ALIReal[ItoF]

Utility of Indian sender and French receiver with the ALIReal model for ItoF treatment and for small $\beta$ it's represented in Figure 5. Symmetrically, utility of French sender and Indian receiver with the ALIReal model for FtoI treatment and again for small $\beta$ it's represented in Figure 6. The smallest amount that a French receiver prefers to its reservation utility is then higher for the ItoF treatment than the one an Indian receiver accepts for the FtoI treatment. The ALIReal model shows several interesting qualitative features. For the ItoF treatment, in Figure 5, the predicted equilibrium is far from the equal split of the pie in real terms (i.e. $2 \$$ for the Indian sender and $8 \$$ for the French receiver) and, in this case, it is quite close to the fifty-fifty share. In other words, the amount the French receiver should get is significantly lower than the fair amount he could expect in a fair share in real terms. Things are different for the FtoI treatment. There, in Figure 6, the predicted equilibrium for the game with the ALIReal model is close to the fair share in real term. The Indian receiver's outcome at equilibrium is not significantly different from this fair share. 


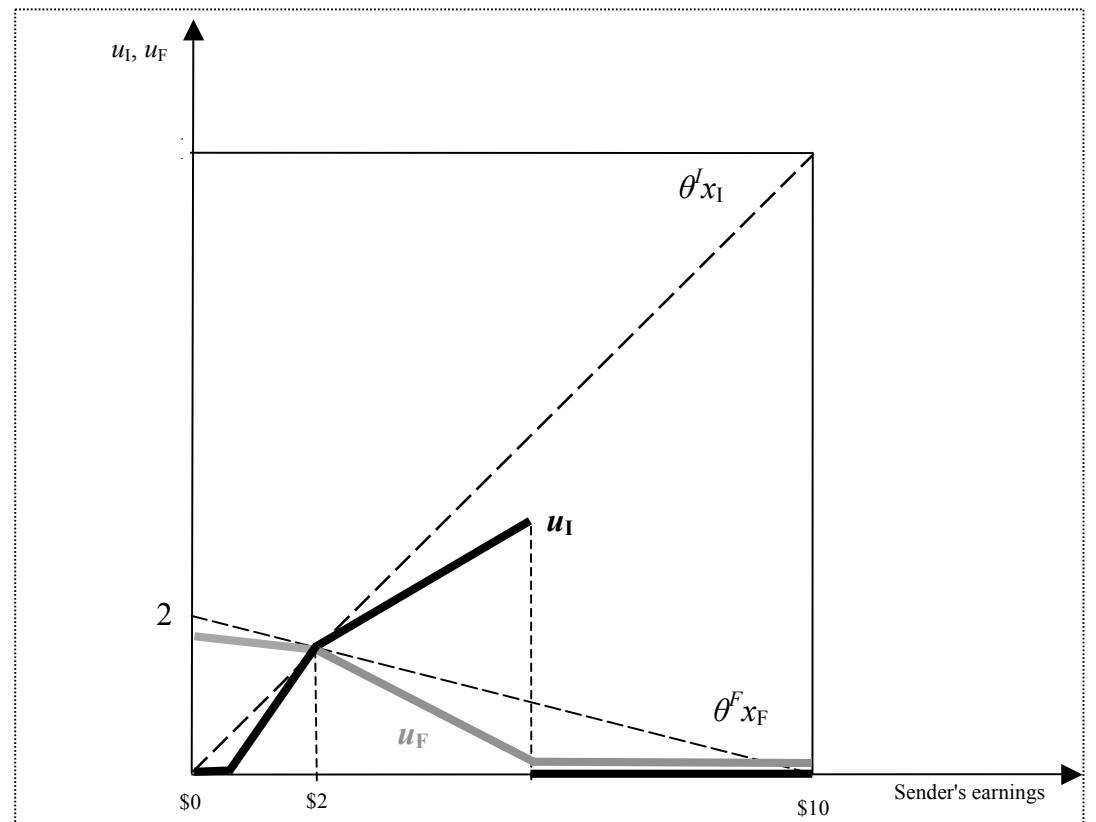

Figure 5. Utility of Indian sender and French receiver with the ALFReat moder ror Itö treatment and for small $\beta$

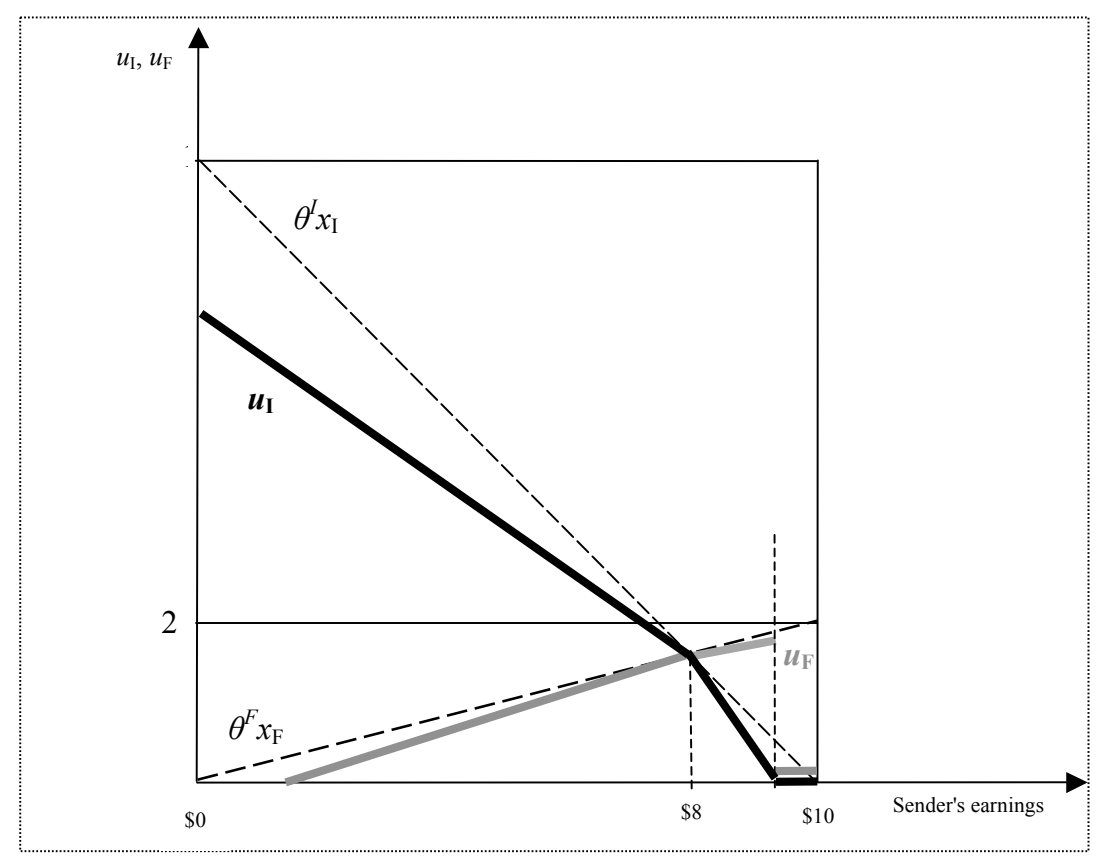


The predictions of the ALIReal model are in accordance with our findings. We do not observe a significant difference between the FtoF and FtoI treatments. On the other hand, the amount required by Indian receiver is lower for FtoI treatment and the amount acceptable for a French receiver is higher for ItoF treatment.

(3) The AGINom models.

These models predict that the Indians senders propose nothing to French receivers. This is obviously not in line with our data.

(4) The AGIReal models.

These models do not match observations in the trans-continental treatments, for the following reason. With the relative specification of inequity aversion, the inequity terms are at their maximum ( $\alpha$ and $\beta$ ) independently of the player's actions. They thus disappear in the solving of the game and the equilibrium prediction is (both in ItoF and FtoI interactions) that the sender keeps (almost) everything. This clearly contradicts our findings. With the absolute specification of inequity aversion, the prediction that Indian senders offer almost nothing to French receivers again contradict the observation.

We conclude that the present experiment refutes models of aversion to inequality based on Global or Nominal considerations. Models based on the aversion to Local inequality in Real terms are not refuted, in particular the model of Aversion to Local Inequity in Real terms.

\section{Discussion}

An important question in a multi-country setting is whether individuals belonging to different societies have different preferences for equity according to their culture. If one thinks that the only difference between this Transcontinental UG and a standard UG is that subjects have different norms of fairness due to their culture, the Fehr-Schmidt model can be assumed and the $\alpha$ and $\beta$ parameters interpreted as tastes for equity specific to each country. This extended model would thus have 4 parameters rather than 2. But, is this required to explain players' behavior? Our observations indicate there is no need to multiply the number 
of parameters and that, instead, the original Schmidt's model suffices, once the nature of payoffs involved in the negotiation is taken into consideration.

There exist two reasons for which avoiding culture-specific models it is attractive. First, if norms of culture are specific to countries or societies, one should be able to observe them in the normal framing of intra-country ultimatum game. That is, as we have observed that Indian made quite substantial offers and reject only very low offers, we could extrapolate such a behavior and conclude that Indian are highly averse to unfair distributional outcomes as senders and lowly averse to unfair split as receivers. But if this was true, Indians should be equally highly/lowly averse to unfairness when they play against Indians. In other words, if a population effect exists and it is relevant, we should observe Indians making high offers to Indians and Indian rejecting low offers made by Indians with a low propensity. As we have discussed above, one can easily reject the first fact, although it is harder to conclude on the second. On the French side, the FtoF treatment shows that French senders behave as their Slovenian or American fellows (Roth et al. 1991), for instance, they will offer nearly the equal split in most of French-French interactions and reject more than one time in two an offer lower than $20 \%$ of the stake. Once again, this is not what we observed when French are confronted with Indians (at least, as far as concerns the sender's behavior). Our intra-country offer data does not contradict the findings of Oosterbeek et al. (2004) in their meta-analysis of UG research on cultural differences.

The second reason why models that detail the nature of the payoffs are important is the fact that it can be used to interpret a larger set of laboratory data, and in particular not only the data coming from transcontinental experimentation. Consider the experiment by Kagel, Kim and Moser, where senders and receivers were alternatively applied different rule for computing their final payoff. In the protocol, they specify a value of $\theta$ nearly equal to 3 . With such a value and considering the same value for $\alpha(\alpha=1 / 4)$, our model can organize their observations: the estimated rejection threshold is about $37.5 \%$ of the stake when the sender is given the low conversion rate and $6 \%$ when the sender is given the high conversion rate (and $\beta$ varies between $1 / 4$ and $3 / 4^{24}$ ).

Finally we have to discuss the possibility that bargaining behavior changes across time. A plausible explanation is that a sort of dynamic effect operates complementarily to the Local 
Equity norm. The specific norm of fairness that is implemented in a transcontinental ultimatum game needs some time to be operational and effective. Both players are likely to spend the first rounds of the game to partially adjust their behavior on their opponent's one, as they need to improve their reciprocal knowledge; the discovering of the other is accompanied by the implementation of the relevant norm of fairness.

The transcontinental protocol allows to distinguish between two factors: 1) the ex-ante status of the player and 2) the game status of the players. Usually these two elements are confounded as players are equal ex-ante: they are drawn from the same population and a) by implicit assumption, they are endowed with the same preferences - in particular with identical marginal utility of money - and b) by the means of experimental protocol, they have the same initial endowments. While in the traditional $\mathrm{UG}$, the inequity aversion concerns can only intervene with respect to the "unjust structure" of the bargaining game, in our version of the UG players might make use of the game to impose a social norm that might re-establish the equality.

Our findings are relevant for understanding which kind of norms of Justice are internalized by the individuals. The agents can consider interactive situations in two different ways. According to a first conception of Justice, the interaction is one small world within which equity norms apply. The interpersonal comparisons of utility are here performed at the level of marginal utilities involved in the experiment. We call "Local Equity" this conception. For instance, Local Equity could sustain the argument that an equitable division is one such that each participant can buy the same amount of good with the marginal income of the experiment. The crucial point is here that one can buy more with 5 dollars in India than in France.

According to a second conception of Justice, an interaction is an opportunity to modify the situation of the individuals in some "just" direction. The interpersonal comparisons of utility are here performed at the level of non-marginal utilities, that is utility taking into account the individual's status beyond the experiment, for instance her total income. Call this the "Compensation Equity" conception. Compensation could sustain the argument that an equitable division is one such that the amount of goods that participants can buy with their total income tends to be equalized through the experiment. The crucial point is then that only

\footnotetext{
${ }^{24}$ The average offers for the first and second treatment were respectively of 54.4 and 24.2 out of 100 .
} 
allocating more to the Indians than to the French will go in the direction of equalizing total incomes. Our data show that Local Equity, rather than Compensation Equity, is the relevant conception of Justice for explaining the subjects' behavior.

We therefore reach, in the bargaining context, the same conclusion as Elster (1991) in other contexts: “...doctors and other specialist allocators do not see their role as that of reducing social injustice. They are specialized providers of specific services, not promoters of overall welfare. (...) If the specialists are aware that there is a bigger picture, they leave it to others. Often, however, nobody feels responsible for the bigger picture. The many localjustice decisions that are made by different institutions can add up to a global injustice." 


\section{References}

Andreoni, James and Miller, John, 2002. Giving According to GARP: An Experimental Test of the Consistency of Preferences for Altruism. Econometrica, 70(2), 737-753.

Bolton, Gary E, 1991. A Comparative Model of Bargaining: Theory and Evidence. The American Economic Review, 81(5), 1097-1136.

Bolton, Gary E and Ockenfels, Axel, 2000. ERC: A Theory of Equity, Reciprocity, and Competition. The American Economic Review, 90(1), 166-193.

Buchan, Nancy R., Croson, Rachel T.A. and Johnson, Eric J., 1997, Trust and reciprocity: An international experiment, Working Paper.

Camerer, Colin F., 2003, Behavioral Game Theory: Experiments in Strategic Interaction. Princeton University Press (Princeton) and Russell Sage Foundation (New York).

Charness, Gary and Rabin, Matthew, 2002. Understanding Social Preferences with Simple Tests. Quarterly Journal of Economics, 117(3), 817-869.

Dufwenberg, Martin and Kirchsteiger, Georg, 2004. A theory of sequential reciprocity. Games and Economic Behavior, 47(2), 268-298.

Elster, Jon, 1991, Local justice and interpersonal comparisons. In: Jon Elster and John E. Roemer (Eds.), Interpersonal Comparisons of Well-Being,. Cambridge University Press, pp. 98-126.

Elster, Jon, 1992, Local Justice: How institutions allocate scarce goods and necessary burdens. Russel Sage Foundation.

Fehr, Ernst and Schmidt, Klaus, 1999. A Theory of Fairness, Competition, and Cooperation. Quarterly Journal of Economics, 114(August), 769-816.

Gintis, Herbert, 2000, Game theory evolving. Princeton University Press. 
Güth, Werner, 1995. On ultimatum bargaining experiments: a personal review. Journal of Economic Behavior and Organization(27), 329-344.

Güth, Werner, R., R., Schmittberger and B., Schwarz, 1982. An experimental analysis of ultimatum bargaining. Journal of Economic Behavior \& Organization(3), 367-388.

Henrich, Joseph, Boyd, Robert, Bowles, Samuel, Colin Camerer, Fehr, Ernst et al., 2001. In Search of Homo Economicus: Behavioral Experiments in 15 Small-Scale Societies. American Economic Review, 91(2), 73-78.

Kagel, John H., Kim, Chung and Moser, Donald, 1996. Fairness in Ultimatum Games with Asymmetric Information and Asymmetric Payoffs. Games and Economic Behavior, 13(1), 100-110.

Kirchsteiger, Georg, 1994. The role of envy in ultimatum games. Journal of Economic Behavior \& Organization, 25(3), 373-389.

Oosterbeek, Hessel, Sloof, Randolph and van de Kuilen, Gijs, 2004. Cultural Differences in Ultimatum Game Experiments: Evidence from a Meta-Analysis. Experimental Economics, 7(2), 171-188.

Rabin, Matthew, 1993. Incorporating Fairness into Game Theory and Economics. American Economic Review, 83(December), 1281-1302.

Roth, Alvin E., 1995, Bargaining Experiments. In: J. H. Kagel and A.E. Roth (Eds.), Handbook of Experimental Economics. Princeton University Press, Princeton, pp. 253-341.

Roth, Alvin E., Prasnikar, Vesna, Okuno Fujiwara, Masahiro and Zamir, Shmuel, 1991. Bargaining and Market Behavior in Jerusalem, Ljubjana, Pittsburg, and Tokyo: An Experimental Study. American Economic Review, 81(5), 1068-1095.

Sen, Amartya, 1992, Inequality reexamined. Clarendon Press, Oxford. 


\section{Appendix 1. Instruction (ItoF treatment)}

\section{Introduction}

Thank you very much for participating in this experiment. The object of the session is to study how people make decisions. If you follow the instructions and make careful decisions, you might earn a certain amount of money.

\section{Currency}

The currency used in this experiment is US dollars. All monetary amounts will be denominated in this currency. Your earning in dollars will be converted into Rupees at an exchange rate to be described later. Details of how to make decisions and earn money, and of how you will be paid, are provided below.

\section{The decision situation}

In this experiment, you will participate in six rounds. In each round, you will be paired with another person and both of you will be asked to make decisions.

You will never be informed of the identity of any of the people with whom you are paired, nor will any of them be informed of your identity.

In each round you will be presented with a problem about which you must make a decision. In each problem there are two decision makers: a sender and a receiver. You will be assigned either the role of a sender or that of a receiver randomly at the beginning of the experiment. You will keep the same role for all six rounds but will be paired with a different individual in each round.

In this decision-making situation the sender must decide how much of a given amount of dollars, in this case $\$ 10$, to send to the receiver. (Offers must be made in multiples of 0.5 US dollars). The receiver must decide whether to accept or reject the sender's offer. If the receiver accepts the offer, then the receiver gets a payoff equal to the offer and the sender gets a payoff equal to 10 minus the offer. If the receiver rejects the offer, then both the sender and the receiver will get a payoff of 0 . For example: say the sender chooses to offer the receiver $\mathrm{x}$ dollars out of the available ten, if offer is accepted, the sender's payoff will be 10-x and the receiver's payoff will be $\mathrm{x}$, but if the offer is rejected both the sender and the receiver will get 0 . At each round, the sender is paired with a different receiver and he has $\$ 10$ available for the new offer.

The people with whom you will be paired

In this experiment, the other people who participate at the decision problem are French students who have very similar characteristics to you in terms of age, studies and so on. Your decisions will be transmitted via an Internet-Chat Connection, since they will be physically located in France. The experiment co-ordinator will provide the transmission. 


\section{How the experiment takes place}

At the beginning of the experiment, you will be given an envelope. On the back of the envelope you will find your Identification Number (ID). Take care of that number, as you will have to use it throughout the experiment.

In the envelop you will find:

One identification card

Six experiment cards (one for each round)

The identification card tells you if you are to act as receiver or sender.

If you are the sender:

In the first round please take the first round experiment card and write down your offer. Then put the card into the envelope. The experiment assistant will then collect the envelopes. You will have to wait for 5-7 minutes (the time required to transmit your offer to France and receive the answers back) before the experiment can continue. Once the answers have been received, your experiment card will be retuned to you. You will find the receiver's decision to accept or reject your offer as well as your income in that round.

Before starting the next round, the experiment assistant will collect the experiment card. Once you have been told that the second round can begin, repeat the above steps.

If you are the receiver:

In the first round please take the first round experiment card and wait for some minutes while the sender's offer is made and transmitted. The experiment assistant will collect your card and give it back to you with the sender's offer. You will then write down whether you accept or reject the offer, put the card back into the envelope and return it to the experiment assistant. After this your income for the round will be computed and written onto your card which is then returned to you. Before the next round starts, the experiment assistant will collect the experiment card of that round. When you are told that the following round can begin, take a new experiment card and repeat the above steps.

Warning : you are not allowed to communicate with the other participants at any time of experiment. If you do so, you will not receive any payment at the end of the experiment.

After the sixth round...

Between the end of the sixth round and the moment of receiving your payment, you will be given a questionnaire about the experiment. The questionnaire is also part of the experiment and it is important that you fill in every part. The questionnaire is anonymous. You do not have to sign it, nor are you asked to reveal your identity. After completing the questionnaire, the experiment assistant will collect it and accompany you to the payment room. In this room will be a payment envelope with your ID on the back. Show your identification card to the assistant and hand over the experimental materiel you have been delivered with (envelop, pen, instructions sheets) in order to get the envelope. 
How you will get paid

You will receive 2 US dollars simply for showing up today and completing the experiment.

In addition, you will receive a payment based on the outcome of the six rounds of the experiment in which you participated. Two out of the six rounds that you participated in will be randomly chosen and you will receive the payoff that you earned in these two rounds. For instance if rounds 3 and 4 are drawn and your payoffs in those two rounds were $\mathrm{x}$ and $\mathrm{y}$, you will receive $(\mathrm{x}+\mathrm{y}) \mathrm{US} \$+2 \mathrm{US} \$$. The random draw will be done publicly, by using a dice, after the end of the sixth round (there will be two random draws, one for each country).

How your payoff will be converted into cash

The exchange rate that will be used to compute your final payment is the following:

For every dollar that you obtain in the decision problem, you will receive 47 Rupees, which approximately corresponds to the current exchange rate.

The French students will also receive 2 US dollars for their participation. For every dollar they receive in the decision problem, they will receive 1.1 Euro, which approximately corresponds to the Euro-Dollar exchange rate.

To sum-up: both you and French students will receive the following payments: 2US\$ for your participation and the payoff of the two rounds selected by the random draw. All the amounts of money in dollars will be converted into Rupees for you, and into Euros for French Students. 
Some details about the purchasing power in the two different countries

Here there are some details about the purchasing power in France (prices are on average):

1US $\$=1$ coffee in the university campus

2US $\$=1 \mathrm{Mc}$ Donald Cheese-Burger

$5 \mathrm{US} \$=1$ cinema ticket

$8 \mathrm{US} \$=1$ paper-back book (French pocket edition)

20US\$= 1 music-CD (e.g. international rock artist/Bruce Springsteen)

Yearly 2001 GDP per capita in France: 23472 US\$

Here there are some details about the purchasing power in India (prices are on average):

$1 \mathrm{US} \$=1$ cinema ticket

$2 \mathrm{US} \$=1$ meal in a medium class restaurant

$5 \mathrm{US} \$=1$ music-CD (e.g. international rock artist/Bruce Springsteen)

8US\$= 4 English penguin paper-back books

$20 \mathrm{US} \$=$ Fare for a return train journey $(3000 \mathrm{~km}$, i.e. $1500 \mathrm{~km}$ one-way $)$ for 1 person

Yearly 2001 GDP per capita in India: 473 US\$

French students are given the same informations about the purchasing power in the two countries and about the exchange rate of Euro-Dollars and Rupees-Dollars.

Thank you very much for your participation!!! 


\section{Test for understanding}

Please answer the following two control questions:

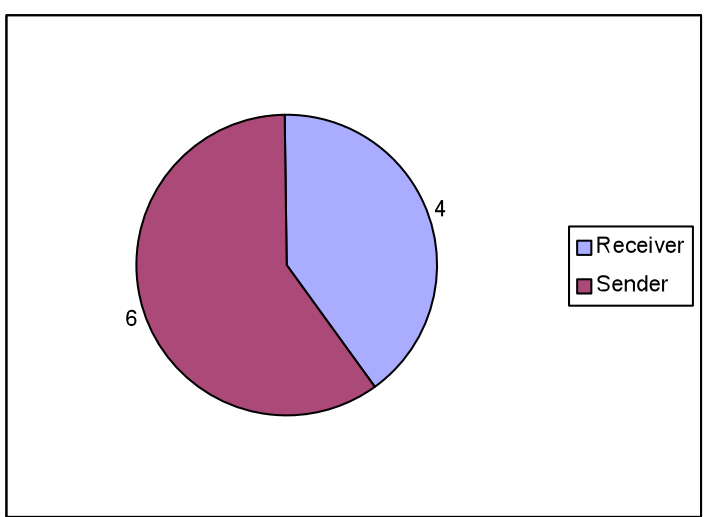

The sender makes an offer to the receiver for the amount of 4 dollars out of the available 10 . The receiver accepts this offer. Thus:

The receiver obtains.

The sender obtains.

The sender makes an offer to the receiver for the amount of 4 dollars out of the available 10 . The receiver rejects this offer. Thus:

The receiver obtains.

The sender obtains. 


\section{Appendix 2. Solving of the games}

Here we find the equilibria of all the relevant bargaining games. We describe the equilibria in terms of the nominal share obtained by the receiver. In order to streamline the discussion, we each time write down in particular the solution if $\beta$ is small. In order to simplify notation, we take one dollar as the monetary unit of the stake to be share, so that $x_{i}$ and $x_{j} \in[0 ; 1]$ and $x_{i}+x_{j}=1$.

\section{Solving the ALINom model.}

The utility is given by the base-line formula (equation 1) of the Fehr-Schmidt model. The utility of player $i$ as a function of her payoff is:

$$
\begin{aligned}
& u_{i}\left(x_{i}\right)=(1+2 \alpha) x_{i}-\alpha \text { if } x_{i} \leq .5 \\
& \text { and } \\
& u_{i}\left(x_{i}\right)=(1-2 \beta) x_{i}+\beta \text { if } x_{i}>.5
\end{aligned}
$$

If $\beta \leq 1 / 2$, the utility is strictly increasing with the payoff. In case of rejection the utility is zero for both players so the (only) sub-game perfect equilibrium of this game is that the sender matches the acceptation threshold of the receiver $x_{j}$ such that $u_{j}\left(x_{j}\right)=(1+2 \alpha) x_{j}-\alpha=0$, that is

$$
x_{j}=\frac{\alpha}{2 \alpha+1}
$$

If $\beta>1 / 2$, the utility is increasing up to $x_{i}=1 / 2$ and decreasing after with a maximum at the equal split $1 / 2$. In that case the (only) sub-game perfect equilibrium of this game is the equal splitting of the pie. The predictions of this model is that outcomes should be the same in all four treatments

\section{Solving the ALIReal model.}

The direct utilities are real payoffs, $y_{i}=\theta_{i} x_{i}$. For Indian player $\theta_{i}=\theta^{I}=1$ then $y_{I}=x_{I}$. For French player $\theta_{i}=\theta^{F}=0.25$ then $y_{F}=x_{F} / 4$. It is necessary to study all four treatments. 


\subsection{Treatment ItoI}

For this treatment, the model is formally identical to the base-line model.

$u_{i}\left(x_{i}, x_{j}\right)=\theta^{I} x_{i}-\alpha\left(\theta^{I} x_{j}-\theta^{I} x_{i}\right)=x_{i}-\alpha\left(x_{j}-x_{i}\right)$ if $x_{i} \leq .5$

and

$u_{i}\left(x_{i}, x_{j}\right)=\theta^{I} x_{i}-\beta\left(\theta^{I} x_{i}-\theta^{I} x_{j}\right)=x_{i}-\beta\left(x_{i}-x_{j}\right)$ if $x_{i}>.5$

The prediction for this treatment is that the receiver receives .5 if $\beta \geq 1 / 2$ and receives $x_{j}=\frac{\alpha}{2 \alpha+1}$ if $\beta<1 / 2$. Let this threshold be denoted:

ALIReal[ItoI] $=\frac{\alpha}{2 \alpha+1}$.

\subsection{Treatment FtoF}

This case is the same as the previous one. For $\beta<1 / 2$ :

ALIReal[FtoF $]=\frac{\alpha}{2 \alpha+1}$.

\subsection{Treatment ItoF}

Let $i$ denote the Indian sender and $j$ denote the French receiver. The point of equal payoffs $y_{i}=y_{j}$ is here $\theta^{I} x_{i}=\theta^{F} x_{i}$, which corresponds to $x_{i}=.2$, and $x_{j}=.8$. For the sender:

$u_{i}\left(x_{i}, x_{j}\right)=\theta^{I} x_{i}-\alpha\left(\theta^{F} x_{j}-\theta^{I} x_{i}\right)=x_{i}-\alpha\left(\frac{x_{j}}{4}-x_{i}\right)=\left(1+\frac{5}{4} \alpha\right) x_{i}-\frac{\alpha}{4}$ if $x_{i} \leq .2$

and

$u_{i}\left(x_{i}, x_{j}\right)=\theta^{I} x_{i}-\beta\left(\theta^{I} x_{i}-\theta^{F} x_{j}\right)=x_{i}-\beta\left(x_{i}-\frac{x_{j}}{4}\right)=\left(1-\frac{5}{4} \beta\right) x_{i}+\frac{\beta}{4}$ if $x_{i}>.2$

and for the receiver :

$u_{j}\left(x_{i}, x_{j}\right)=\theta^{F} x_{j}-\alpha\left(\theta^{I} x_{i}-\theta^{F} x_{j}\right)=\frac{x_{j}}{4}-\alpha\left(x_{i}-\frac{x_{j}}{4}\right)=\left(\frac{1}{4}+\frac{5}{4} \alpha\right) x_{j}-\alpha$ if $x_{j}<.8$ and

$u_{j}\left(x_{i}, x_{j}\right)=\theta^{F} x_{j}-\beta\left(\theta^{F} x_{j}-\theta^{I} x_{i}\right)=\frac{x_{j}}{4}-\beta\left(\frac{x_{j}}{4}-x_{i}\right)=\left(\frac{1}{4}-\frac{5}{4} \beta\right) x_{j}+\beta$ if $x_{j}>.8$ 
If $\beta<4 / 5$, the sender's utility is increasing with her share $x_{i}$ and the receiver's utility is decreasing with $x_{i}$ if $x_{i}>$.2. Consequently the prediction is that if $\beta>4 / 5$, the receiver receives .8 and if $\beta<4 / 5$ she receives:

$$
\text { ALIReal[ItoF] }=\frac{4 \alpha}{5 \alpha+1} .
$$

\subsection{Treatment FtoI}

Let $i$ denote the French sender and $j$ denote the Indian receiver. The point of equal payoff is such that $y_{i}=y_{j}$, that is here $\theta^{F} x_{i}=\theta^{I} x_{i}$, which corresponds to $x_{i}=.8$, and $x_{j}=.2$. For the sender:

$$
\begin{aligned}
& u_{i}\left(x_{i}, x_{j}\right)=\theta^{F} x_{i}-\alpha\left(\theta^{I} x_{j}-\theta^{F} x_{i}\right)=\frac{x_{i}}{4}-\alpha\left(x_{j}-\frac{x_{i}}{4}\right)=\left(\frac{1}{4}+\frac{5}{4} \alpha\right) x_{i}-\alpha \text { if } x_{i} \leq .8 \\
& \text { and } \\
& u_{i}\left(x_{i}, x_{j}\right)=\theta^{F} x_{i}-\beta\left(\theta^{F} x_{i}-\theta^{I} x_{j}\right)=\frac{x_{i}}{4}-\beta\left(\frac{x_{i}}{4}-x_{j}\right)=\left(\frac{1}{4}-\frac{5}{4} \beta\right) x_{i}+\beta \text { if } x_{i}>.8
\end{aligned}
$$

and for the receiver :

$$
\begin{aligned}
& u_{j}\left(x_{i}, x_{j}\right)=\theta^{I} x_{j}-\alpha\left(\theta^{F} x_{i}-\theta^{I} x_{j}\right)=x_{j}-\alpha\left(\frac{x_{i}}{4}-x_{j}\right)=\left(1+\frac{5}{4} \alpha\right) x_{j}-\frac{\alpha}{4} \text { if } x_{j} \leq .2 \\
& \text { and } \\
& u_{j}\left(x_{i}, x_{j}\right)=\theta^{I} x_{j}-\beta\left(\theta^{I} x_{j}-\theta^{F} x_{i}\right)=x_{j}-\beta\left(x_{j}-\frac{x_{i}}{4}\right)=\left(1-\frac{5}{4} \beta\right) x_{j}+\frac{\beta}{4} \text { if } x_{j}>.2
\end{aligned}
$$

If $\beta<1 / 5$, the sender's utility is increasing with her share $x_{i}$ and the receiver's utility is decreasing with $x_{i}$ if $x_{i}>.8$. Consequently the prediction is that the receiver receives .2 if $\beta>1 / 5$ and receives:

ALIReal[FtoI] $=\frac{\alpha}{5 \alpha+4}$ if $\beta<1 / 5$.

\section{Solving the AGINom model.}

Here $y_{i}=R_{i}+x_{i}$.

\subsection{Treatment ItoI}


$u_{i}\left(x_{i}, x_{j}\right)=R^{I}+x_{i}-\alpha\left(x_{j}-x_{i}\right)=R^{I}+(1-2 \alpha) x_{i}-\alpha$ if $x_{i} \leq .5$

and

$u_{i}\left(x_{i}, x_{j}\right)=R^{I}+x_{i}-\beta\left(x_{i}-x_{j}\right)=R^{I}+(1+2 \beta) x_{i}-\beta$ if $x_{i}>.5$

The utility in case of rejection is $R^{I}$. One can see that, in that case, the pre-game wealth cancels and the prediction is similar to the baseline model: the receiver receives .5 if $\beta>1 / 2$ and receives $x_{j}=\frac{\alpha}{2 \alpha+1}$ if $\beta<1 / 2$. We denote:

AGINom[ItoI] $=\frac{\alpha}{2 \alpha+1}$.

\subsection{Treatment FtoF}

This case is the same as the previous one. For $\beta<1 / 2$ :

$A G I N o m[F t o F]=\frac{\alpha}{2 \alpha+1}$.

\subsection{Treatment ItoF}

Because of our hypothesis on the wealth levels, the difference $R^{F}+x_{j}-R^{I}-x_{i}$ is positive, therefore the utility of the Indian sender $i$ is:

$u_{i}\left(x_{i}, x_{j}\right)=R^{I}+x_{i}-\alpha\left(R^{F}+x_{j}-R^{I}-x_{i}\right)=(1+2 \alpha) x_{i}-\alpha R^{F}+(1-\alpha) R^{I}-\alpha$

increasing with her share. Likewise the utility of the French receiver $j$ is

$u_{j}\left(x_{i}, x_{j}\right)=R^{F}+x_{j}-\beta\left(R^{F}+x_{j}-R^{I}-x_{i}\right)=(1-2 \beta) x_{j}+(1-\beta) R^{F}+\beta R^{I}+\beta$

and her utility in case of rejection is $(1-\beta) R^{F}+\beta R^{I}$. It follows that for $\beta>1 / 2$ the model predicts that the receiver (paradoxically) accepts only offers such that $(1-2 \beta) x_{j}+\beta>0$, that is $x_{j}<\frac{\beta}{2 \beta-1}$. The equilibrium is that the Indian sender offers nothing. For $\beta<1 / 2$ the prediction is also that the French receiver accepts any offer and the Indian sender keeps everything 
AbAGINom [ItoF] $=0$.

\subsection{Treatment FtoI}

Because of our hypothesis on the wealth levels, the difference $R^{F}+x_{j}-R^{I}-x_{i}$ is positive, therefore the utility of the French sender $i$ is:

$$
u_{i}\left(x_{i}, x_{j}\right)=R^{F}+x_{i}-\beta\left(R^{F}+x_{i}-R^{I}-x_{j}\right)=(1-2 \beta) x_{i}+\beta+(1-\beta) R^{F}+\beta R^{I}
$$

which is increasing if $\beta<1 / 2$ and decreasing if not. Likewise the utility of the Indian receiver $j$ is:

$$
u_{j}\left(x_{i}, x_{j}\right)=R^{I}+x_{j}-\alpha\left(R^{F}+x_{i}-R^{I}-x_{j}\right)=(1+2 \alpha) x_{j}-\alpha-\alpha R^{F}+(1-\alpha) R^{I}
$$

and her utility in case of rejection is $-\alpha R^{F}+(1-\alpha) R^{I}$. If $\beta>1 / 2$, the equilibrium is that the French sender offers everything and the Indian receiver accepts. If $\beta<1 / 2$, the equilibrium is at the threshold

$$
\text { AbAGINom }[\text { FtoI }]=\frac{\alpha}{2 \alpha+1}
$$

4. Solving the AGIReal model.

Here $y_{i}=\theta_{i}\left(R_{i}+x_{i}\right)$.

\subsection{Treatment ItoI}

The model is formally identical to the baseline model. The prediction is again that the receiver receives .5 if $\beta>1 / 2$ and receives $x_{j}=\frac{\alpha}{2 \alpha+1}$ if $\beta<1 / 2$.

We let: $A$ GIReal[ItoI] $=\frac{\alpha}{2 \alpha+1}$.

\subsection{Treatment FtoF}


$u_{i}\left(x_{j}, x_{i}\right)=\theta^{F} R^{F}+\theta^{F} x_{i}-\alpha \theta^{F}\left(x_{j}-x_{i}\right)=\frac{1}{4}\left(R^{F}+(1+2 \alpha) x_{i}-\alpha\right)$ if $x_{i} \leq .5$

and

$u_{i}\left(x_{j}, x_{i}\right)=\theta^{F} R^{F}+\theta^{F} x_{i}-\beta \theta^{F}\left(x_{i}-x_{j}\right)=\frac{1}{4}\left(R^{F}+(1-2 \beta) x_{i}+\beta\right)$ if $x_{i}>.5$

The utility in case of rejection is $\frac{1}{4} R^{F}$. One can see that, in that case, the pre-game wealth cancels and the prediction is similar to the baseline model: the receiver receives .5 if $\beta>1 / 2$ and receives $x_{j}=\frac{\alpha}{2 \alpha+1}$ if $\beta<1 / 2$. So we note:

$$
\text { AGIReal }[F t o F]=\frac{\alpha}{2 \alpha+1} .
$$

\subsection{Treatment ItoF}

For the Indian sender:

$$
u_{i}\left(x_{j}, x_{i}\right)=\theta^{I} R^{I}+\theta^{I} x_{i}-\alpha\left(\theta^{F} R^{F}+\theta^{F} x_{j}-\theta^{I} R^{I}-\theta^{I} x_{i}\right)=R^{I}-\frac{\alpha}{4} R^{F}+\alpha R^{I}+\left(1+\frac{5 \alpha}{4}\right) x_{i}-\frac{\alpha}{4}
$$

The Indian sender's utility is increasing with $x_{i}$.

For the French receiver :

$$
u_{j}\left(x_{j}, x_{i}\right)=\theta^{F} R^{F}+\theta^{F} x_{j}-\beta\left(\theta^{F} R^{F}+\theta^{F} x_{j}-\theta^{I} R^{I}-\theta^{I} x_{i}\right)=\frac{1-\beta}{4} R^{F}+\beta R^{I}+\left(\frac{1}{4}-\frac{5 \beta}{4}\right) x_{j}+\beta
$$

and the receiver utility in case of rejection is $\frac{1-\beta}{4} R^{F}+\beta R^{I}$. The receiver accepts the offer if $\frac{1-5 \beta}{4} x_{j}+\beta>0$. If $\beta>1 / 5$, the receiver (paradoxically) accepts only offers smaller than $\frac{4 \beta}{5 \beta-1}$ and thus the equilibrium is that the Indian sender keeps everything for her. If $\beta<1 / 5$, the equilibrium is also that the Indian sender keeps everything for her :

AGIReal[ItoF] $=0$.

\subsection{Treatments FtoI}

For the French sender: 


$$
u_{i}\left(x_{i}, x_{j}\right)=\theta^{F} R^{F}+\theta^{F} x_{i}-\beta\left(\theta^{F} R^{F}+\theta^{F} x_{i}-\theta^{I} R^{I}-\theta^{I} x_{j}\right)=\frac{1-\beta}{4} R^{F}+\beta R^{I}+\left(\frac{1}{4}-\frac{5 \beta}{4}\right) x_{i}+\beta
$$

is increasing with $x_{i}$ if $\beta<1 / 5$ and decreasing otherwise.

For the Indian receiver:

$u_{j}\left(x_{i}, x_{j}\right)=\theta^{I} R^{I}+\theta^{I} x_{j}-\alpha\left(\theta^{F} R^{F}+\theta^{F} x_{j}-\theta^{I} R^{I}-\theta^{I} x_{i}\right)=-\alpha \theta^{F} R^{F}+(1+\alpha) \theta^{I} R^{I}+\left(\theta^{I}-\alpha\left(\theta^{I}-\theta^{F}\right) x_{j}+\alpha \theta^{I}\right.$ and the receiver utility in case of rejection is $-\alpha \theta^{F} R^{F}+(1+\alpha) \theta^{I} R^{I}$.

Note that $\theta^{I}-\alpha\left(\theta^{I}-\theta^{F}\right)>0$, thus if $\beta>1 / 5$ the equilibrium is that the Indian receives everything and if $\beta<1 / 5$, the equilibrium is that the Indian receives nothing:

AGIReal[FtoI] $=0$. 